\title{
Commutators of Pseudodifferential Operators on Weighted Hardy Spaces
}

\author{
Yu-long Deng $(\mathbb{D}$ \\ School of Mathematics Science, Changsha Normal University, Changsha 410100, China \\ Correspondence should be addressed to Yu-long Deng; yuldeng@163.com
}

Received 29 September 2021; Accepted 22 December 2021; Published 20 January 2022

Academic Editor: Antonio Masiello

Copyright (c) 2022 Yu-long Deng. This is an open access article distributed under the Creative Commons Attribution License, which permits unrestricted use, distribution, and reproduction in any medium, provided the original work is properly cited.

In this paper, we establish an endpoint estimate for the commutator, $[b, T]$, of a class of pseudodifferential operators $T$ with symbols in Hörmander class $S_{\rho, \delta}^{m}\left(\mathbb{R}^{n}\right)$. In particular, there exists a nontrivial subspace of $B M O\left(\mathbb{R}^{n}\right)$ such that, when $b$ belongs to this subspace, the commutators $[b, T]$ is bounded from $H_{\omega}^{1}\left(\mathbb{R}^{n}\right)$ into $L_{\omega}^{1}\left(\mathbb{R}^{n}\right)$, which we extend the well-known result of CalderónZygmund operators.

\section{Introduction}

The purpose of this paper is to find out a proper subspace of $B M O\left(\mathbb{R}^{n}\right)$ such that, the commutators of pseudodifferential operators $T$ is bounded on weighted Hardy space $H_{\omega}^{1}\left(\mathbb{R}^{n}\right)$, where the operators $T$ associated with the symbols in the Hölmander class $S_{\rho, \delta}^{m}\left(\mathbb{R}^{n}\right)$ and $\omega \in A_{p}\left(\mathbb{R}^{n}\right)$. As in [1], we firstly recall some notations and lemmas. For $m \in \mathbb{R}$ and $\rho, \delta \in[0,1]$, a symbol $a(x, \xi) \in S_{\rho, \delta}^{m}\left(\mathbb{R}^{n}\right)$ is a smooth function defined on $\mathbb{R}^{n} \times \mathbb{R}^{n}$ such that

$$
\left|\partial_{x}^{\alpha} \partial_{\xi}^{\beta} a(x, \xi)\right| \leq C_{\alpha, \beta}(1+|\xi|)^{m-\rho|\beta|+\delta|\alpha|},
$$

holds for all multi-indices $\alpha, \beta \in \mathbb{N}^{n}$, where $C_{\alpha, \beta}$ is independent of $x$ and $\xi$ (see, e.g., [2]).

Given an infinitely differentiable function $f \in \mathbb{R}^{n}$ with compact supports and symbol $a(x, \xi) \in S_{\rho, \delta}^{m}\left(\mathbb{R}^{n}\right)$, the pseudodifferential operator $T$ is defined by

$$
T f(x)=\int_{\mathbb{R}^{n}} a(x, \xi) \mathrm{e}^{2 \pi i x \cdot \xi} \hat{f}(\xi) \mathrm{d} \xi,
$$

where $\hat{f}$ is the Fourier transform of $f$ and we write $T \in \mathscr{L}_{\rho, \delta}^{m}$ . Moreover, the operator $T$ can be expressed by a distribution kernel $K(x, y)$ as (see, e.g., [3])

$$
T f(x)=\int K(x, y) f(y) \mathrm{d} y .
$$

Let $b \in B M O\left(\mathbb{R}^{n}\right)$ and $T$ be a Calderón-Zygmund operator. A classical result in [4] stated that the commutator operators $[b, T]$, defined by

$$
[b, T] f(x)=b(x) T f(x)-T(b f)(x),
$$

is bounded on $L^{p}\left(\mathbb{R}^{n}\right)$ for $p \in(1, \infty)$. However, it fails to be of weak type $(1,1)$ and of type $\left(H^{1}\left(\mathbb{R}^{n}\right), L^{1}\left(\mathbb{R}^{n}\right)\right)$ when $b \in B M O\left(\mathbb{R}^{n}\right)$ (see, $\left.[5,6]\right)$. Instead, some endpoint theories are provided.

Remark that if the symbol $a(x, \xi)$ satisfies some particular assumptions, pseudodifferential operator $T$ in $\mathscr{L}_{\rho, \delta}^{m}$ is a Calderón-Zygmund operator (see, [7]). Correspondingly, when $b \in B M O\left(\mathbb{R}^{n}\right)$ and $T \in \mathscr{L}_{\rho, \delta}^{m}$, the boundness of $[b, T]$ on Lebesgue space $L^{p}\left(\mathbb{R}^{n}\right)$ for $p \in(1, \infty)$ was considered (see, e.g., $[8-10])$.

It is widely known that $H^{1}\left(\mathbb{R}^{n}\right)$ is an advantageous substitute for $L^{1}\left(\mathbb{R}^{n}\right)$. The behavior of commutator $[b, T]$ on $H^{1}\left(\mathbb{R}^{n}\right)$ has also attracted a lot of interest. For example, when $b \in L M O_{\infty}\left(\mathbb{R}^{n}\right)$ (see, [11]), Yang et al. [12] obtained that $[b, T]$ is bounded from $H^{1}\left(\mathbb{R}^{n}\right)$ into $L^{1}\left(\mathbb{R}^{n}\right)$, where $T \in \mathscr{L}_{1, \delta}^{0}$ with $\delta \in[0,1)$; Hung and Ky [13] established an estimate for $[b, T]$ on local Hardy space $h^{1}\left(\mathbb{R}^{n}\right)$. Very recently, Deng and Long [14] got an estimate for $[b, T]$ from $H^{1}\left(\mathbb{R}^{n}\right)$ into weak $L^{1}\left(\mathbb{R}^{n}\right)$, where $b \in B M O\left(\mathbb{R}^{n}\right)$.

For $\omega \in A_{p}\left(\mathbb{R}^{n}\right)$, there are numerous papers dealing with the weighted $L^{p}$ boundedness of the commutators $[b, T]$ for 
$p \in(1, \infty)$ and we refer to [15-20] for more details, where $T \in \mathscr{L}_{\rho, \delta}^{m}$ and $b \in B M O\left(\mathbb{R}^{n}\right)$. A nature question is that can one establish an estimate for $[b, T]$ on weighted Hardy spaces $H_{\omega}^{1}\left(\mathbb{R}^{n}\right)$ ?

In general, the commutators $[b, T]$ is not bounded from the weighted Hardy spaces $H_{\omega}^{1}\left(\mathbb{R}^{n}\right)$ into the weighted Lebesgue spaces $L_{\omega}^{1}\left(\mathbb{R}^{n}\right)$ if $b \in B M O\left(\mathbb{R}^{n}\right)$ is not a constant function, even $T$ is a Calderón-Zygmund operator. It is worthy to pointing out that in [21], Liang et al. found a proper subspaces of $B M O\left(\mathbb{R}^{n}\right)$, such that, the commutators of Calderón-Zygmund operator is bounded on weighted Hardy spaces. Motivated by this result, we wonder whether there exists a nontrivial subspace of $B M O\left(\mathbb{R}^{n}\right)$ such that when $b$ belongs to this subspace, the commutators $[b, T]$ of pseudodifferential operator is bounded on $H_{\omega}^{1}\left(\mathbb{R}^{n}\right)$.

The main concern of this paper is to give an answer to the above question. For this purpose, we recall the definition of the Muckenhoupt weights $A_{p}\left(\mathbb{R}^{n}\right)$. A nonnegative measurable function $\omega$ is said to be in the Muckenhoupt class $A_{p}\left(\mathbb{R}^{n}\right)$ for $p \in(1, \infty)$, if

$[\omega]_{A_{p}\left(\mathbb{R}^{n}\right)}=\sup _{B \subset \mathbb{R}^{n}}\left(\frac{1}{|B|} \int_{B} \omega(x) \mathrm{d} x\right)\left(\frac{1}{|B|} \int_{B} \omega(x)^{-(1 / p-1)} \mathrm{d} x\right)^{p-1}<\infty$, and for $p=1$, if

$[\omega]_{A_{1}\left(\mathbb{R}^{n}\right)}=\sup _{B \subset \mathbb{R}^{n}}\left(\frac{1}{|B|} \int_{B} \omega(x) \mathrm{d} x\right)\left(\operatorname{esssup}_{x \in B} \omega(x)^{-1}\right)<\infty$,

where the supremum is taken over all balls $B \subset \mathbb{R}^{n}$ and $A_{\infty}\left(\mathbb{R}^{n}\right)=\bigcup_{p \geq 1} A_{p}\left(\mathbb{R}^{n}\right)$.

As known, if $\omega \in A_{p}$, then $\omega \in A_{q}$ for some $q \in[1, p)$. We thus write $q_{\omega}=\inf \left\{p \geq 1: \omega \in A_{p}\right\}$ to denote the critical index of $\omega$. For a measurable set $E$, we denote $\omega(E)=\int_{E} \omega(x) \mathrm{d} x$. The following lemma provides a way to compare $|E|$ and $\omega(E)$ of a set $E$ (see [22]).

Lemma 1. Let $\omega \in A_{p}$ and $p \geq 1$. Then, there exists a constant $C>0$ such that

$$
C\left(\frac{|E|}{|B|}\right)^{p} \leq \frac{\omega(E)}{\omega(B)}
$$

for all balls $B$ and measurable subsets $E \subset B$.

Definition 1. Let $\omega \in A_{\infty}\left(\mathbb{R}^{n}\right)$ and $\int_{\mathbb{R}^{n}} \omega(x) / 1+|x|^{n} \mathrm{~d} x<\infty$. A locally integrable function $b$ is said to belong to $\mathscr{B} \mathscr{M} \sigma_{\omega}\left(\mathbb{R}^{n}\right)$ if

$$
\|b\|_{\mathscr{B} \mathscr{M} \mathcal{O}_{\omega}\left(\mathbb{R}^{n}\right)}=\sup _{B \subset \mathbb{R}^{n}}\left\{\frac{1}{\omega(B)}\left[\int_{\mathbb{R}^{n} \backslash B} \frac{\omega(x)}{\left|x-x_{0}\right|^{n}} \mathrm{~d} x\right]\left[\int_{B}\left|b(y)-b_{B}\right| \mathrm{d} y\right]\right\}<\infty
$$

Here, $b_{B}=(1 /|B|) \int_{B} b(x) \mathrm{d} x$ and the supremum is taken over all balls $B=B\left(x_{0}, r\right) \subset \mathbb{R}^{n}$ with center $x_{0}$ and radius $r$.

We point out that the space $\mathscr{B} \mathscr{M} \mathcal{O}_{\omega}\left(\mathbb{R}^{n}\right)$ has been studied in $[21,23,24]$. A locally integrable function $b$ is said to be in $B M O\left(\mathbb{R}^{n}\right)$ if

$$
\|b\|_{B M O\left(\mathbb{R}^{n}\right)}=\sup _{B \subset \mathbb{R}^{n}} \frac{1}{|B|} \int_{B}\left|b(x)-b_{B}\right| \mathrm{d} x<\infty
$$

where the supremum is taken over all balls $B \subset \mathbb{R}^{n}$.

In [21], the space $\mathscr{B} \mathscr{M} \mathcal{O}_{\omega}\left(\mathbb{R}^{n}\right)$ is proved to be a subspace of $B M O\left(\mathbb{R}^{n}\right)$, and not be a trivial space since it contains the Lipschitz function with compact support and also that,

Lemma 2. Let $\omega \in A_{\infty}\left(\mathbb{R}^{n}\right)$ and $p \in[1, \infty)$. Then, there exists a constant $C>0$ such that, for any $f \in B M O\left(\mathbb{R}^{n}\right)$ and any ball $B \subset \mathbb{R}^{n}$,

$$
\left(\frac{1}{\omega(B)} \int_{B}\left|f(x)-f_{B}\right|^{p} \omega(x) \mathrm{d} x\right)^{1 / p} \lesssim\|f\|_{B M O\left(\mathbb{R}^{n}\right)}
$$

The first main result is stated as follow.

Theorem 1. Let $\epsilon=\min \{1,(1+m+n) / \rho\}, \quad \omega \in A_{1+(\epsilon / n)}$ satisfies $\int_{\mathbb{R}^{n}} \omega(x) / 1+|x|^{n} \mathrm{~d} x<\infty$ and $b \in \mathscr{B} \mathscr{M O}_{\omega}\left(\mathbb{R}^{n}\right)$. Assume that the pseudodifferential operator $T \in \mathscr{L}_{\rho, \delta}^{m}$ with $\rho \in(0,1], \delta \in[0,1)$ and

$$
m \in(-(n+1),-(n+1)(1-\rho)] .
$$

Then, the commutator $[b, T]$ is bounded from $H_{\omega}^{1}\left(\mathbb{R}^{n}\right)$ into $L_{\omega}^{1}\left(\mathbb{R}^{n}\right)$; i.e., there exists a constant $C>0$ such that, for all $f \in H_{\omega}^{1}\left(\mathbb{R}^{n}\right)$,

$$
\|[b, T] f\|_{L_{\omega}^{1}\left(\mathbb{R}^{n}\right)} \leq C\|b\|_{\mathscr{B}, \mathscr{M} \mathcal{O}_{\omega}\left(\mathbb{R}^{n}\right)} .
$$

Finally, we make some conventions on notations. $C$ denotes a positive constant may change from line to line and we write $a \leq b$ as shorthand for $a \leq C b$. If $a \leq b$ and $b \leq a$, we mean $a \sim b$. For a measurable set $A,|A|$ denotes the Lebesgue measure of $A$. $B$ will always denote a ball and $t B(t>0)$ denotes the ball $B$ dilated by $t$.

\section{Notations and Technical Lemmas}

In this section, we begin our story by presenting an estimate about the pseudodifferential operator $T$ associated with the kernel $K(x, y)$. Let $\delta\left(\mathbb{R}^{n}\right)$ be the class of Schwartz functions and $\mathcal{S}^{\prime}\left(\mathbb{R}^{n}\right)$ be its dual space. The space of $C^{\infty}$-function with compact support is denoted by $C_{0}^{\infty}\left(\mathbb{R}^{n}\right)$. Pseudodifferential operators are bounded from $\mathcal{S}\left(\mathbb{R}^{n}\right)$ to $\mathcal{S}\left(\mathbb{R}^{n}\right)$ and so possess distribution kernels $K(x, y) \in \mathcal{S}^{\prime}\left(\mathbb{R}^{n} \times \mathbb{R}^{n}\right)$. Then, the following formula for the kernel is useful (cf. Proposition 1 in [25], see also [26]).

Lemma 3. Let $a(x, \xi) \in \mathcal{S}_{\rho, \delta}^{m}\left(\mathbb{R}^{n}\right)$ with $0<\rho \leq 1,0 \leq \delta<1$ and associate with the pseudodifferential operator $T \in \mathscr{L}_{\rho, \delta}^{m}$. 
Then, the distribution kernel $K(x, y)$ of $T$ is smooth away from the diagonal $\left\{(x, x): x \in \mathbb{R}^{n}\right\}$ and is given by

$$
K(x, y)=\lim _{\varepsilon \longrightarrow 0} \int_{\mathbb{R}^{n}} \mathrm{e}^{2 \pi i(x-y) \cdot \xi} a(x, \xi) \psi(\varepsilon \xi) \mathrm{d} \xi,
$$

where $\psi \in C_{0}^{\infty}\left(\mathbb{R}^{n}\right)$ satisfies $\psi(\xi)=1$ for $|\xi| \leq 1$ and the limit is taken in $\mathcal{S}^{\prime}\left(\mathbb{R}^{n}\right)$ and independent of the choice of $\psi$. If $M \in \mathbb{N}$ and $M+m+n>0, K(x, y)$ satisfies the estimates

$$
\sup _{|\alpha+\beta|} D_{x}^{\alpha} D_{y}^{\beta} K(x, y) \mid \leq C_{M} \frac{1}{|x-y|^{(M+m+n) / \rho}}, x \neq y .
$$

Moreover, for any multi-index $\alpha, \beta \in \mathbb{N}^{n}$ and $N \in \mathbb{N}$,

$$
\sup _{|x-y| \geq 1 / 2}|x-y|^{N}\left|D_{x}^{\alpha} D_{y}^{\beta} K(x, y)\right| \leq C_{\alpha, \beta, N} .
$$

In [20], the following is derived from Lemma 3.

Lemma 4. Let $\rho \in(0,1], \delta \in[0,1)$ and $m \in(-(n+1)$, $-(n+1)(1-\rho)]$ and the pseudodifferential operator $T \in \mathscr{L}_{\rho, \delta}^{m}$ associated with the distribution kernel $K(x, y)$. Then, for any $y \in B=B\left(x_{0}, r\right)$ and every $x \in 2^{j+1} B \backslash 2^{j} B$, we have

$$
\left|K(x, y)-K\left(x, x_{0}\right)\right| \leqslant 2^{-j(n+\varepsilon)} r^{-j n},
$$

where $\epsilon=\min \{1,1+n+m / \rho\}$.

Let $\omega \in A_{\infty}\left(\mathbb{R}^{n}\right)$ and $p \in(0, \infty)$. We denote by $L_{\omega}^{p}\left(\mathbb{R}^{n}\right)$ the weighted Lebesgue space of all measurable functions $f$ satisfying

$$
\|f\|_{L_{\omega}^{p}\left(\mathbb{R}^{n}\right)}=\left(\int_{\mathbb{R}^{n}}|f(x)|^{p} \omega(x) \mathrm{d} x\right)^{1 / p}<\infty .
$$

When $p=\infty, L_{\omega}^{\infty}\left(\mathbb{R}^{n}\right)$ is defined to be the same as $L^{\infty}\left(\mathbb{R}^{n}\right)$, the following useful $L_{\omega}^{p}$ bounds for pseudodifferential operator $T \in \mathscr{L}_{\rho, \delta}^{m}$ are due to Michalowski et al. [15].

Lemma 5. Let $T \in \mathscr{L}_{\rho, \delta}^{m}$ with $\rho \in(0,1], \delta \in[0,1)$ and $m \in(-\infty,-n(1-\rho))$. Then, for each $p \in(1, \infty)$ and $\omega \in A_{p}$, there exists a constant $C>0$ such that

$$
\left\|\left(b-b_{B}\right) T a\right\|_{L_{\omega}^{1}\left(\mathbb{R}^{n}\right)} \leq C\|b\|_{B M O\left(\mathbb{R}^{n}\right)}
$$

Let $\phi \in \mathcal{S}\left(\mathbb{R}^{n}\right)$ with $\int \phi \mathrm{d} x \neq 0$. Then, for any $x \in \mathbb{R}^{n}$, the maximal function of a distribution $f \in \mathcal{S}^{\prime}\left(\mathbb{R}^{n}\right)$ is defined by

$$
\phi^{*}(f)(x)=\sup _{t>0}\left|\phi_{t}^{*} f(x)\right|
$$

where $\phi_{t}(y)=\left(1 / t^{n}\right) \phi(y / t)$ for any $t>0$. Let $p \in(1, \infty)$. Then, the maximal function is bounded on $L_{\omega}^{p}\left(\mathbb{R}^{n}\right)$ if and only if $\omega \in A_{p}$. Analogous to the classical Hardy space, the weighted Hardy space $H_{\omega}^{1}\left(\mathbb{R}^{n}\right)$ can be defined in terms of maximal functions.

Definition 2. Let $\omega \in A_{\infty}$. The weighted Hardy space $H_{\omega}^{1}\left(\mathbb{R}^{n}\right)$ is defined by

$H_{\omega}^{1}\left(\mathbb{R}^{n}\right)=\left\{f \in \mathcal{S}^{\prime}\left(\mathbb{R}^{n}\right): \varphi^{*}(f)(x)=\sup _{t>0}\left|\varphi_{t} * f(x)\right| \in L_{\omega}^{1}\left(\mathbb{R}^{n}\right)\right\}$, which is independent of the choice of $\varphi \in \mathcal{S}\left(\mathbb{R}^{n}\right)$. Moreover, we define $\|f\|_{H_{\omega}^{1}\left(\mathbb{R}^{n}\right)}=\left\|\varphi^{*}(f)\right\|_{L_{\omega}^{1}\left(\mathbb{R}^{n}\right)}$.

Definition 3. Let $\omega$ be a weight with critical index $q_{\omega}$. $A_{n}$ $(\omega, 1, \infty)$-atom is a function $a$ satisfying

$$
\operatorname{supp}(a) \subset B, \| a_{\| L^{\infty}\left(\mathbb{R}^{n}\right)} \leq \omega(B)^{-1},
$$

and $\int_{\mathbb{R}^{n}} a(x) x^{\alpha} \mathrm{d} x=0$ for every multi-index $\alpha$ with $|\alpha| \leq\left[n\left(q_{\omega}-1\right)\right] . \quad$ Conventionally, $\quad T^{*} 1=0 \quad$ means $\int_{\mathbb{R}^{n}} \operatorname{Ta}(x) \mathrm{d} x=0$ for all $(\omega, 1, \infty)$-atoms $a$.

The Hardy space $H_{\omega}^{1}\left(\mathbb{R}^{n}\right)$ is spanned by all of $(\omega, 1, \infty)$-atoms (see [22]). Namely,

$$
f=\sum_{j} \lambda_{j} a_{j}
$$

in the sense of $\mathcal{S}^{\prime}$, where each $a_{j}$ is an $(\omega, 1, \infty)$-atom and $\lambda_{j}$ satisfies

$$
\sum_{j}\left|\lambda_{j}\right|<\infty
$$

Moreover, $\|f\|_{H_{\omega}^{1}\left(\mathbb{R}^{n}\right)}=\inf \left\{\sum_{j=1}^{\infty}\left|\lambda_{j}\right|: f=\sum_{j=1}^{\infty} \lambda_{j} a_{j}\right\}$.

Deng et al. [1] got some sufficient conditions for the boundedness of pseudodifferential operators $T \in \mathscr{L}_{\rho, \delta}^{m}$ on weighted Hardy space $H_{\omega}^{1}\left(\mathbb{R}^{n}\right)$.

Lemma 6. Let $\epsilon=\min \{1,1+m+n / \rho\}, \quad p \in[1,1+\epsilon / n)$, $\omega \in A_{p}$, and $T \in \mathscr{L}_{\rho, \delta}^{m}$ with $\rho \in(0,1], \quad \delta \in[0,1)$. If $m \in(-(n+1),-(n+1)(1-\rho)]$, then $T$ is bounded from $H_{\omega}^{1}\left(\mathbb{R}^{n}\right)$ into $L_{\omega}^{1}\left(\mathbb{R}^{n}\right)$, i.e., there exists a constant $C>0$ such that

$$
\|T f\|_{L_{\omega}^{1}\left(\mathbb{R}^{n}\right)} \leq C\|f\|_{H_{\omega}^{1}\left(\mathbb{R}^{n}\right)} .
$$

\section{The Proof of Theorem 1}

In this section, we establish the sufficient condition for the boundedness of $[b, T]$ from $H_{\omega}^{1}\left(\mathbb{R}^{n}\right)$ into $L_{\omega}^{1}\left(\mathbb{R}^{n}\right)$. As in [21], we need the following proposition.

Proposition 1. Write $\epsilon=\min \{1,(1+m+n) / \rho\}$. Let $p \in(1,1+(\epsilon / n))$ and $\omega \in A_{p}\left(\mathbb{R}^{n}\right)$. Assume that the pseudodifferential operator $T \in \mathscr{L}_{\rho, \delta}^{m}$ with $\rho \in(0,1], \delta \in[0,1)$ and $m \in(-(n+1),-(n+1)(1-\rho)]$. Then, there exists a constant $C>0$ such that, for any $b \in B M O\left(\mathbb{R}^{n}\right)$ and $(\omega, 1, \infty)$-atom $a$,

$$
\left\|\left(b-b_{B}\right) T a\right\|_{L_{\omega}^{1}\left(\mathbb{R}^{n}\right)} \leq C\|b\|_{B M O\left(\mathbb{R}^{n}\right)},
$$

where $\operatorname{supp}(a) \subset B=B\left(x_{0}, r\right)$.

Proof. It suffices to show that

$$
I_{1}=\int_{2 B}\left|b\left(x-b_{B}\right) T a(x)\right| \omega(x) \mathrm{d} x \leq C\|b\|_{B M O\left(\mathbb{R}^{n}\right)},
$$

and 
$\left.I_{2}=\int_{(2 B)^{6}} b(x)-b_{B}\right) T a(x) \mid \omega(x) \mathrm{d} x \leq C\|b\|_{B M O\left(\mathbb{R}^{n}\right)}$.
For $I_{1}$, it is easy to see that

$$
\begin{aligned}
I_{1} & \leq \int_{2 B}\left|b_{2 B}-b_{B}\right||T a(x)| \omega(x) \mathrm{d} x+\int_{2 B}\left|b(x)-b_{2 B} \| T a(x)\right| \omega(x) \mathrm{d} x \\
& \leq\left|b_{2 B}-b_{B}\right|\|T a\|_{L_{\omega}^{1}\left(\mathbb{R}^{n}\right)}+\int_{2 B}\left|b(x)-b_{2 B} \| T a(x)\right| \omega(x) \mathrm{d} x \\
& =I_{11}+I_{12} .
\end{aligned}
$$

Then, by Lemma 6, the boundedness of the operator $T$ from $H_{\omega}^{1}\left(\mathbb{R}^{n}\right)$ to $L_{\omega}^{1}\left(\mathbb{R}^{n}\right)$, we conclude that

$$
I_{11} \leq C\|b\|_{B M O\left(\mathbb{R}^{n}\right)} .
$$

$$
\begin{aligned}
I_{12} & \leq\left(\int_{2 B}\left|b(x)-b_{2 B}\right|^{q^{\prime}} \omega(x) \mathrm{d} x\right)^{1 / q^{\prime}}\left(\int_{2 B}|T a(x)|^{q} \omega(x) \mathrm{d} x\right)^{1 / q} \\
& \leq C(\omega(2 B))^{1-(1 / q)}\|b\|_{B M O\left(\mathbb{R}^{n}\right)}\|T a\|_{L_{\omega}^{q}\left(\mathbb{R}^{n}\right)} \\
& \leq C(\omega(2 B))^{1-(1 / q)}\|b\|_{B M O\left(\mathbb{R}^{n}\right)}\|a\|_{L_{\omega}^{q}\left(\mathbb{R}^{n}\right)} \\
& \leq C\|b\|_{B M O\left(\mathbb{R}^{n}\right)}
\end{aligned}
$$

Thus, (26) holds.

For $I_{2}$, by the moment condition of $(\omega, 1, \infty)$-atoms $a$, we have

$$
\begin{aligned}
I_{2} & =\int_{(2 B)^{\complement}}\left|b(x)-b_{B}\right|\left|\int_{B} a(y)\left[K(x, y)-K\left(x, x_{0}\right)\right] \mathrm{d} y\right| \omega(x) \mathrm{d} x \\
& \leq \int_{B}|a(y)| \int_{(2 B)^{\complement}}\left|b(x)-b_{B} \| K(x, y)-K\left(x, x_{0}\right)\right| \omega(x) \mathrm{d} x \mathrm{~d} y \\
& =\int_{B}|a(y)| \sum_{k=1}^{\infty} \int_{2^{k+1} B \backslash 2^{k} B}\left|b(x)-b_{B} \| K(x, y)-K\left(x, x_{0}\right)\right| \omega(x) \mathrm{d} x \mathrm{~d} y .
\end{aligned}
$$

Then, we apply Lemma 4 to get

$$
\begin{aligned}
I_{2} & \leq C \int_{B}|a(y)| \sum_{k=1}^{\infty} \int_{2^{k+1} B \backslash 2^{k} B} 2^{-k(n+\epsilon)} r^{-k n}\left|b(x)-b_{B}\right| \omega(x) \mathrm{d} x \mathrm{~d} y \\
& \leqslant \frac{|B|}{\omega(B)} \sum_{k=1}^{\infty} \frac{2^{-k \epsilon}}{\left|2^{k+1} B\right|} \int_{2^{k+1} B}\left|b(x)-b_{B}\right| \omega(x) \mathrm{d} x .
\end{aligned}
$$

Finally, by using Lemma 2 again and combing the $\quad\left|b_{2^{k+1} B}-b_{B}\right| \leq \sum_{j=0}^{k}\left|b_{2^{j+1} B}-b_{2^{j} B}\right| \lesssim(k+1)\|b\|_{B M O\left(\mathbb{R}^{n}\right)}$,
equality 
with Lemma 1 and the condition $p \in(1,1+(\epsilon / n))$, we deduce

$$
\begin{aligned}
I_{2} & \leq C \frac{|B|}{\omega(B)} \sum_{k=1}^{\infty} 2^{-k \varepsilon} k \frac{\omega\left(2^{k+1} B\right)}{\left|2^{k+1} B\right|}\|b\|_{B M O\left(\mathbb{R}^{n}\right)} \\
& \leq C\|b\|_{B M O}\left(\mathbb{R}^{n}\right) \sum_{k=1}^{\infty} k 2^{-k(\varepsilon+n-p n)} \leq C\|b\|_{B M O\left(\mathbb{R}^{n}\right)},
\end{aligned}
$$

and which suggests that (27) holds. Thus, we finish the proof of Proposition 1.

Now, we are ready to give the proofs of Theorem 1.

Proof. Write $\epsilon=\min \{1,(1+m+n) / \rho\}$. Let $\omega \in A_{1+(\epsilon / n)}$ and $f \in H_{\omega}^{1}\left(\mathbb{R}^{n}\right)$. According to the atomic characterization of $f$ and Proposition 1, it is reduced to showing that

$$
\left\|T\left(\left(b-b_{B}\right) a\right)\right\|_{L_{\omega}^{1}\left(\mathbb{R}^{n}\right)} \leq C\|b\|_{\mathscr{B} \mathscr{M} \mathcal{O}_{\omega}\left(\mathbb{R}^{n}\right)}
$$

holds for each $(\omega, 1, \infty)$-atom $a$ related to some ball $B=B\left(x_{0}, r\right)$.
Then, by the boundedness of $T$ from $H_{\omega}^{1}\left(\mathbb{R}^{n}\right)$ to $L_{\omega}^{1}\left(\mathbb{R}^{n}\right)$ as in Lemma 6, we just need to prove

$$
\left\|\left(b-b_{B}\right) a\right\|_{H_{\omega}^{1}\left(\mathbb{R}^{n}\right)} \leq\|b\|_{\mathscr{B} \mathscr{M} \sigma_{\omega}\left(\mathbb{R}^{n}\right)} .
$$

Finally, (36) is equivalent to establishing

$$
\left\|\phi^{*}\left(\left(b-b_{B}\right) a\right)\right\|_{L_{\omega}^{1}\left(\mathbb{R}^{n}\right)} \lesssim\|b\|_{\mathscr{B} \mathscr{M} \mathcal{O}_{\omega}\left(\mathbb{R}^{n}\right)},
$$

for $\quad \varphi \in \mathcal{S}\left(\mathbb{R}^{n}\right) \quad$ with $\quad \int \varphi \mathrm{d} x \neq 0$, since $\left\|\left(b-b_{B}\right) a\right\|_{H_{\omega}^{1}\left(\mathbb{R}^{n}\right)}=\left\|\varphi^{*}\left(\left(b-b_{B}\right) a\right)\right\|_{L_{\omega}^{1}\left(\mathbb{R}^{n}\right)}$ as in Definition 2.

In order to get (37), we consider

$$
\begin{aligned}
& I_{3}=\int_{2 B} \phi^{*}\left(\left(b-b_{B}\right) a\right)(x) \omega(x) \mathrm{d} x, \\
& I_{4}=\int_{(2 B)^{\complement}} \phi^{*}\left(\left(b-b_{B}\right) a\right)(x) \omega(x) \mathrm{d} x .
\end{aligned}
$$

For $I_{3}$, combining Hölder's inequality with the weighted $L^{p}$ boundedness of the maximal function and Lemma 2, we have

$$
\begin{aligned}
I_{3} & \leq(\omega(2 B))^{1 / q^{\prime}}\left\|\phi^{*}\left(\left(b-b_{B}\right) a\right)_{\| L_{\omega}^{q}\left(\mathbb{R}^{n}\right)} \leq(\omega(2 B))^{1 / q^{\prime}}\right\|\left(b-b_{B}\right) a_{\| L_{\omega}^{q}\left(\mathbb{R}^{n}\right)} \\
& \leq C\left(\frac{1}{\omega(B)} \int_{B}\left|b(x)-b_{B}\right|^{q} \omega(x) \mathrm{d} x\right)^{1 / q} \leq C\|b\|_{B M O\left(\mathbb{R}^{n}\right)} \\
& \leq\|b\|_{\mathscr{B} \mathscr{M} \mathcal{O}_{\omega}\left(\mathbb{R}^{n}\right)} .
\end{aligned}
$$

For $I_{4}$, noting that $|x-y| \sim\left|x-x_{0}\right|$ for every $x \in(2 B)^{\complement}$

and any $y \in B$, we get

$$
\begin{aligned}
\phi^{*}\left(\left(b-b_{B}\right) a\right) & =\sup _{t>0} \frac{1}{t^{n}} \int_{B}\left|b(y)-b_{B} \| a(y)\right|\left|\phi\left(\frac{x-y}{t}\right)\right| \mathrm{d} y \\
& \lesssim \frac{1}{\left|x-x_{0}\right|^{n}} \int_{B}\left|b(y)-b_{B} \| a(y)\right| \mathrm{d} y .
\end{aligned}
$$

Hence, $I_{4} \leqslant\|b\|_{\mathscr{B} M \mathcal{O}_{\omega}\left(\mathbb{R}^{n}\right)}$ and it completes the proof of Theorem 1.

\section{Data Availability}

The author confirms that no data were used to support this study. All references used were listed.

\section{Conflicts of Interest}

The author declares that he has no conflicts of interest.

\section{Acknowledgments}

The author would like to express his thanks to Xiangtan University for part of the work completed here during his study period. This work was supported by Changsha Normal
University, and the article processing charge is sponsored by her. This study was also supported by the Key Scientic Research Projects of Hunan Education Department (21A0617).

\section{References}

[1] Y. Deng and S. Long, "Pseudodifferential operators on weighted Hardy spaces," Journal of Function Spaces, vol. 2020, Article ID 7154125, 7 pages, 2020.

[2] L. Hörmander, "Pseudo-differential operators and hypoelliptic equations," Proceedings of Symposia in Pure Mathematics, vol. 10, pp. 138-183, 1967.

[3] E. M. Stein, Harmonic Analysis: Real Variable Methods, Orthogonality and Oscillatory Integrals, Princeton University Press, Princeton, NJ, USA, 1993.

[4] R. R. Coifman, R. Rochberg, and G. Weiss, "Factorization theorems for Hardy spaces in several variables," Annals of Mathematics, vol. 103, no. 2, pp. 611-635, 1976. 
[5] L. D. Ky, "Bilinear decompositions and commutators of singular integral operators," Transactions of the American Mathematical Society, vol. 365, no. 6, pp. 2931-2958, 2013.

[6] C. Pérez, "Endpoint estimates for commutators of singular integral operators," Journal of Functional Analysis, vol. 128, pp. 163-185, 1995.

[7] J. Journé, "Calderón-Zygmund operators, pseudo-differential operators and the Cauchy integral of Calderón," Lecture Notes in Mathematics, Vol. 994, Springer-Verlag, , Berlin, Germany, 1983.

[8] P. Auscher and M. E. Taylor, "Paradifferential operators and commutator estimates," Communications in Partial Differential Equations, vol. 20, no. 9-10, pp. 1743-1775, 1995.

[9] S. Chanillo, "Remarks on commutators of pseudo-differential operators," Multidimensional Complex Analysis and Partial Differential Equations, vol. 205, pp. 33-37, 1997.

[10] Y. Lin, "Commutators of pseudo-differential operators," Science in China, Series A: Mathematics, vol. 51, no. 3, pp. 453-460, 2008.

[11] B. Bongioanni, E. Harboure, and O. Salinas, "Commutators of riesz transforms related to schrödinger operators," Journal of Fourier Analysis and Applications, vol. 17, no. 1, pp. 115-134, 2011.

[12] J. Yang, Y. Wang, and W. Chen, "Endpoint estimates for the commutator of pseudo-differential operators," Acta Mathematica Scientia, vol. 34, no. 2, pp. 387-393, 2014

[13] H. D. Hung and L. D. Ky, "An Hardy estimate for commutators of pseudo-differential operators," Taiwanese Journal of Mathematics, vol. 19, no. 4, pp. 1097-1109, 2015.

[14] Y. Deng and S. Long, "Endpoint estimates for commutators of pseudodifferential operators," Acta Mathematica Sinica, Chinese Series, vol. 34, no. 2, pp. 387-393, 2021.

[15] N. Michalowski, D. J. Rule, and W. Staubach, "Weighted norm inequalities for pseudo-pseudodifferential operators defined by amplitudes," Journal of Functional Analysis, vol. 258 , no. 12 , pp. 4183-4209, 2010.

[16] L. Tang, "Weighted norm inequalities for pseudo-differential operators with smooth symbols and their commutators," Journal of Functional Analysis, vol. 262, no. 4, pp. 1603-1629, 2012.

[17] T. A. Bui, "New weighted norm inequalities for pseudodifferential operators and their commutators," International Journal of Analysis, vol. 2013, Article ID 798528, 12 pages, 2013.

[18] S. Nishigaki, "Weighted norm inequalities for certain pseudodifferential operators," Tokyo Journal of Mathematics, vol. 7, pp. 129-140, 1984.

[19] K. Yabuta, "Weighted norm inequalities for pseudo-differential operators," Osaka Journal of Mathematics, vol. 23, pp. 703-723, 1986.

[20] Y. Deng, Z. Chen, and S. Long, "Double weighted commutators theorem for pseudo-differential operators with smooth symbols," Czechoslovak Mathematical Journal, vol. 71, no. 146, pp. 703-723, 2021.

[21] Y. Liang, L. D. Ky, and D. Yang, "Weighted endpoint estimates for commutators of Calderón-Zygmund operators," Proceedings of the American Mathematical Society, vol. 144, no. 12, pp. 5171-5181, 2016.

[22] J. García-Cuerva and J. L. Rubio De Francia, Weighted Norm Inequalities and Related Topics, North-Holland Publishing, Amsterdam, Netherlands, 1985.

[23] S. Bloom, "Pointwise multipliers of weighted BMO spaces," Proceedings of the American Mathematical Society, vol. 105, no. 4, p. 950, 1989.
[24] K. Yabuta, "Pointwise multipliers of weighted BMO spaces," Proceedings of the American Mathematical Society, vol. 117, no. 3, pp. 737-744, 1993.

[25] J. Hounie and R. A. d. S. Kapp, "Pseudodifferential operators on local Hardy spaces," Journal of Fourier Analysis and Applications, vol. 15, no. 2, pp. 153-178, 2009.

[26] J. Alvarez and J. Hounie, "Estimates for the kernel and continuity properties of pseudo-differential operators," Arkiv for Matematik, vol. 28, no. 1, pp. 1-22, 1990. 